\title{
Effets des facteurs abiotiques sur la répartition spatiale des groupements végétaux dans la zone de transition soudano-guinéenne du Bénin
}

\author{
Ismaïla TOKO IMOROU \\ Laboratoire de Cartographie, Département de Géographie et Aménagement du Territoire, Faculté des Lettres \\ Arts et Sciences Humaines, Université d'Abomey-Calavi, 10BP1082 Cotonou-Houéyiho, Bénin \\ Auteur correspondant ; Email : ismael_toko@yahoo.fr, Tel :00 22997772872
}

\section{RESUME}

Les effets des facteurs abiotiques sur la répartition spatiale des groupements végétaux ont été étudiés dans la zone de transition soudano-guinéenne du Bénin. Les relevés phytosociologiques ont été réalisés le long des toposéquences dans 156 placeaux de $900 \mathrm{~m}^{2}$. Le logiciel PC ORD 5.0 a servi à l'ordination des groupements (DCA), à l'analyse discriminante des groupements suivant la topographie et les propriétés du sol (CCA) et à l'identification des espèces caractéristiques (IVA). L'indice de Jaccard est déterminé par le logiciel CAP. Au total, 430 espèces réparties en 257 genres et 78 familles ont été recensées. Huit groupements végétaux répartis suivant un gradient pédologique et topographique sont obtenus après l'ordination. L'analyse discriminante des groupements indique que la profondeur du sol est le facteur le plus déterminant dans la distribution des groupements végétaux. Les groupements végétaux établis au bas des versants sur des sols profonds et humides sont nettement distincts de ceux établis sur les interfluves et à mi-pente sur des sols exondés. L'indice de Jaccard discrimine les groupements, et, la richesse spécifique augmente avec la profondeur du sol, tandis qu'elle baisse avec l'augmentation de la pente et de la pression anthropique. L'indice de Shannon et l'équitabilité de Pielou sont très faibles dans les champs et les dépressions marécageuses dominées par les herbacées. La densité et la surface terrière des ligneux varient respectivement de 104 tiges/ha et $3,21 \mathrm{~m}^{2} /$ ha dans les champs à 1007 tiges/ha et $18,01 \mathrm{~m}^{2} /$ ha au bas des versants et à mi-pente sur des sols profonds et humides. Le biovolume de la strate herbacée varie de $1917 \mathrm{~m}^{3} / \mathrm{ha}$ dans les champs à $15105 \mathrm{~m}^{3} / \mathrm{ha}$ dans les dépressions.

(c) 2013 International Formulae Group. All rights reserved.

Mots clés : groupements végétaux, facteurs abiotiques, répartition spatiale, bassin versant, Bénin.

\section{INTRODUCTION}

La distribution géographique des communautés végétales est régie par des facteurs biotiques et abiotiques et leur discrimination atteste les relations entre la végétation et les facteurs climatiques, édaphiques, topographiques et humains (Russel-Smith, 1991 ; Parmentier et al., 2005 ;
Toko et al., 2010 ; Toko Imorou et al., 2010 ). Sous la dépendance d'un même climat, le relief et le sol ainsi que l'action de l'homme constituent les déterminants de la distribution des groupements végétaux à travers le paysage (Tchamie et Bouraïma 1997). En zone soudano-guinéenne, plusieurs facteurs 
déterminent la répartition spatiale des groupements végétaux.

Au Bénin, malgré les nombreuses études réalisées dans la zone soudanoguinéenne sur les groupements végétaux (Sinsin, 1994 ; Sinsin et al., 1996 ; Oumorou, 1998; Sinsin, 1999; Sinsin et Oumorou, 2000 ; Houinato et Sinsin, 2001; Sinsin, 2001 ; Sinsin et Wotto, 2003 ; Orthmann, 2005 ; Orekan, 2007 ; Toko et al., 2010 ; Toko Imorou et al., 2010), certaines interrogations demeurent, concernant les facteurs déterminant la répartition spatiale des groupements végétaux en considérant les bassins versants comme unités d'analyse. Plus spécifiquement, ces études n'ont pas abordé la structure et le fonctionnement de la végétation à l'échelle des bassins versants. Aussi les facteurs déterminant la variabilité du fonctionnement de la végétation sont-ils abordés isolement alors que l'ensemble des processus mis en jeu dans ce fonctionnement devrait être pris en compte de façon intégrée à l'échelle des bassins versants. L'analyse de la variabilité de la structure et du fonctionnement de la végétation à l'échelle des bassins versants permet de prendre en compte la diversité des conditions édaphiques et topographiques.

La présente étude a pour objectif de déterminer les effets de la topographie et des conditions édaphiques sur la répartition spatiale des groupements végétaux dans les petits bassins versants en zone de transition soudano-guinéenne du Bénin.

\section{MATERIEL ET METHODES Cadre de l'étude}

Les données ont été collectées dans le bassin supérieur du fleuve Ouémé au centre du Bénin entre $8^{\circ}$ et $9^{\circ} 50$ de latitude nord et entre $2^{\circ}$ et $2^{\circ} 50$ de longitude est. Il est dans la zone de transition soudano-guinéenne et est à cheval entre les districts phytogéographiques de Bassila, du Borgou-Nord et du Borgou-Sud (Figure 1). Le secteur est sous l'influence du climat soudano-guinéen. La pluviosité et la température moyenne annuelle sont respectivement de $1150 \mathrm{~mm}$ et de $27{ }^{\circ} \mathrm{C}$. Le substratum géologique dominant dans la région est constitué de formations cristallines très anciennes de type "Dahomeyen". Les sols sont en majorité des sols ferrugineux tropicaux et accessoirement des sols ferrallitiques dans la partie Nord-Ouest (Faure, 1977). Le paysage est essentiellement constitué de pénéplaine cristalline d'altitude moyenne de $300 \mathrm{~m}$. Le relief est dominé par une série d'inselbergs orientés nord-sud dont le plus élevé est le mont Soubakpérou (620 $\mathrm{m})$. Le réseau hydrographique est constitué par plusieurs rivières dont les plus importantes sont l'Ouémé et la Térou. Les principaux types de formations végétales rencontrées sont: les galeries forestières, les forêts claires, les savanes boisées, les savanes arborées, les savanes arbustives et les mosaïques de champs et de jachères.

\section{Méthodes \\ Choix des petits bassins versants et des placeaux}

Les principaux critères de choix des petits bassins versants sont l'importance et la densité du réseau hydrographique, la longueur des versants, l'accessibilité des sites, la présence d'aire protégée et le degré d'anthropisation. $\mathrm{Au}$ total, quatre petits bassins versants à l'intérieur desquels plusieurs transects transversaux (1 à $6 \mathrm{~km}$ ) allant du bas de versant vers le sommet, sont choisis dans le but de recouper la diversité des conditions de sol et de topographie. Des placeaux sont installés suivant les variations de la toposéquence et des faciès de végétation. Pour ressortir 1'influence des conditions de drainage du sol, quelques placeaux sont installés suivant le regard de drainage dans les drains. Le nombre de transects et le nombre de placeaux par petit bassin versant varient selon la complexité de la topographie et de la physionomie de la végétation.

\section{Collecte des données floristiques}

Les relevés phytosociologiques sont effectués suivant la méthode sigmatiste de Braun-Blanquet (Weber et al., 2000). L'aire 
de relevé retenue s'est basée sur les travaux effectués en milieu tropical par plusieurs auteurs (Sinsin, 1999; Oumorou, 2003 ; Wala, 2004 ; Djego et Sinsin, 2006) qui ont utilisé des surfaces variant entre 100 et 1000 $\mathrm{m}^{2}$ selon les formations végétales et les strates. Dans la présente étude, l'aire de relevé est de $100 \mathrm{~m}^{2}(10 \mathrm{~m} \times 10 \mathrm{~m})$ pour la strate herbacée et de $900 \mathrm{~m}^{2}$ (30 m x $\left.30 \mathrm{~m}\right)$ pour les strates arbustive et arborée. La nomenclature utilisée est celle de Hutchinson et Dalziel (1954-1972) et de Lebrun et Storck (19911997).

Les données dendrométriques ont concerné le nombre d'individus de chaque espèce et le diamètre des ligneux de $\mathrm{dbh} \geq 10$ $\mathrm{cm}$.

L'identification des types biologiques des espèces a été réalisée sur la base des travaux de Raunkiaer (1934).

Les types phytogéographiques des espèces sont établis à partir des subdivisions chorologiques de White (1983).

\section{Collecte des données topographiques et pédologiques \\ Les différentes positions} topographiques (dépression, bas de versant, mi-versant et interfluve) sont directement notées sur le terrain. La pente des placeaux est prise au centre, à l'aide du clisimètre.

La texture (sable, limon et argile) des sols est déterminée sur des échantillons de sol prélevés dans les 10 premiers $\mathrm{cm}$ du sol dans les quatre coins et au centre de chaque placeau.

La profondeur des sols est mesurée dans des fosses pédologiques de $50 \mathrm{~cm}$ de côté et d'au moins $1 \mathrm{~m}$ de profondeur.

\section{Traitement des données}

L'ordination des relevés par la Detrended Canonical Analysis (DCA) du logiciel PC ORD version 5 (McCune et Mefford, 1999), a permis d'individualiser les groupements végétaux.

Une analyse canonique des correspondances (CCA) a été faite pour déterminer les relations entre les groupements végétaux identifiés et les propriétés du sol et de la topographie. Pour ce faire, le logiciel PC ORD version 5 a été utilisé.

Le logiciel Indicator Species Analysis (Dufrene et Legendre, 1997) du logiciel PC ORD version 5 a permis de déterminer les espèces caractéristiques de chaque groupement végétal.

Le test de Monte Carlo a permis de mesurer le degré de significativité du caractère indicateur de chaque espèce dans le groupe de relevés auquel elle appartient. Toutes les espèces ayant une probabilité (p) inférieure à 0,05 ont été retenues comme espèces caractéristiques du groupement.

La diversité alpha a été analysée à partir de la richesse spécifique (R), de l'indice de diversité de Shannon (1949) et de l'équitabilité de Pielou (1966).

L'indice de diversité de Shannon $(\mathrm{H})$ a pour formule : $\mathrm{H}=-\Sigma \mathrm{P}_{\mathrm{i}} \log _{2} \mathrm{P}_{\mathrm{i}}$ où $\mathrm{P}_{\mathrm{i}}=\left(\mathrm{n}_{\mathrm{i}} /\right.$ $\mathrm{N})$ est la fréquence relative des individus de l'espèce (i).

L'équitabilité de Pielou (E) a pour formule : $\mathrm{E}=\mathrm{H} / \log _{2} \mathrm{R}$ où $\mathrm{H}$ représente l'indice de Shannon, et $\log _{2} \mathrm{R}$ est la valeur théorique de la diversité maximale pouvant être atteinte dans chaque groupement et $\mathrm{R}$ est la richesse spécifique.

L'indice de similitude de Jaccard (Ij) calculé par le logiciel CAP (Pisces Conservation 2002) a été calculé pour comparer les groupements végétaux. $\mathrm{Si} \mathrm{Ij} \geq 50$ $\%$ alors les deux groupements sont similaires.

Le biovolume $\mathrm{Bv}\left(\mathrm{m}^{3} / \mathrm{ha}\right)$ est calculé selon la formule : $\mathrm{Bv}=\Sigma \mathrm{RM}_{\mathrm{i}} \times \mathrm{H}_{\mathrm{i}} \times 10000 / \mathrm{S}$ où $\mathrm{RM}_{\mathrm{i}}=$ Recouvrement moyen de l'espèce $\mathrm{i}$, obtenu d'après l'échelle d'abondancedominance de Braun-Blanquet (Weber et al., 2000), $H_{i}=$ Hauteur de l'espèce $i(m)$ et 10000/S : superficie inventoriée rapportée à 1'hectare. La densité (D) des ligneux a pour formule : $\mathrm{D}=\mathrm{N}$ x $10000 / \mathrm{S}$ où $\mathrm{D}:$ nombre de tiges/ha ; $\mathrm{N}:$ nombre de tiges de $\mathrm{dbh} \geq 10 \mathrm{~cm}$ et $10000 / \mathrm{S}$ : superficie inventoriée rapportée à 1'hectare. La surface terrière moyenne est calculée selon la formule: $G_{i}=\Sigma \Pi D^{2} \mathrm{x}$ $10000 / 4 S$ où $G_{i}$ est en $\mathrm{m}^{2} /$ ha $; \mathrm{D}$ : diamètre (m) ; 10000/S : superficie inventoriée rapportée à 1 hectare. 


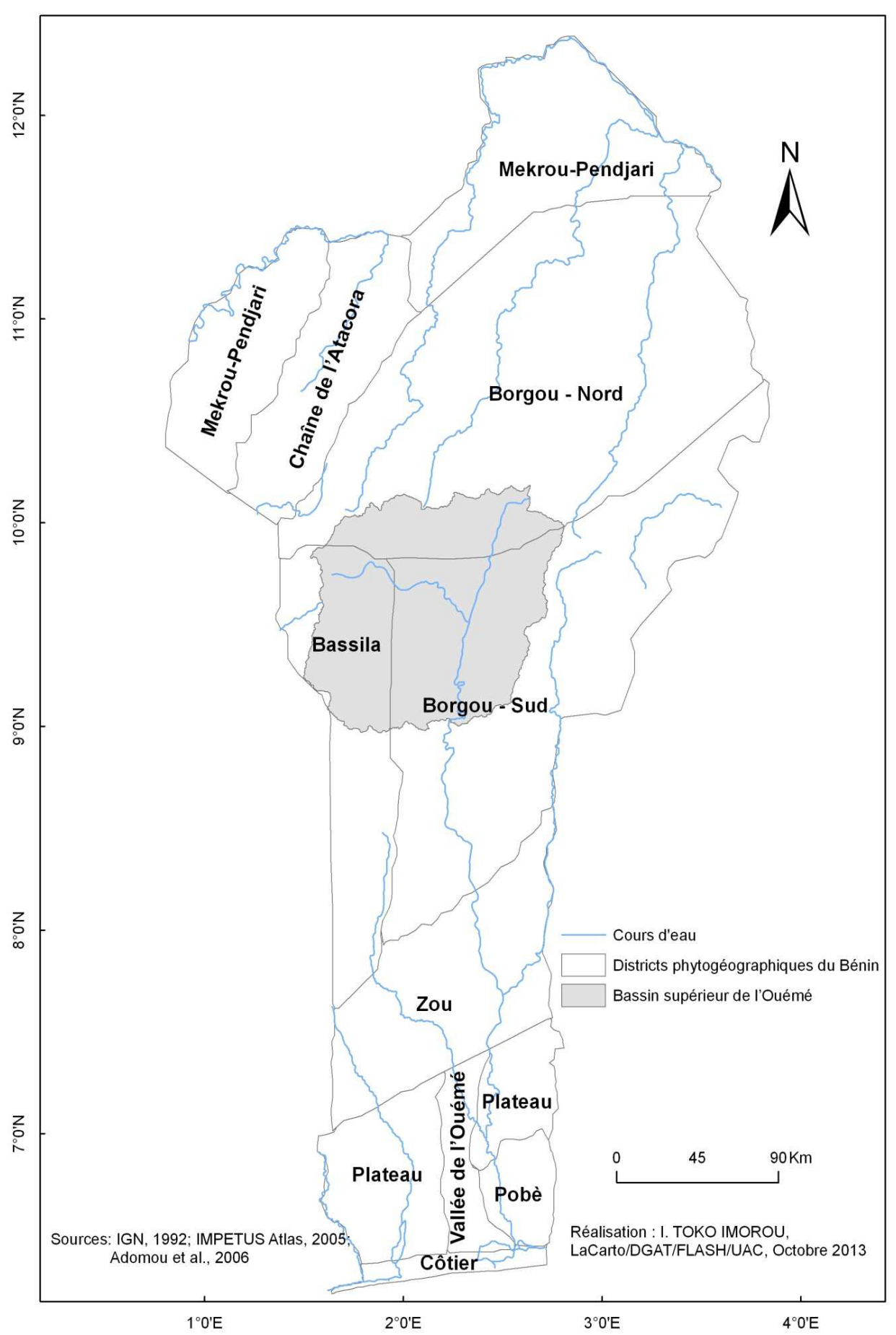

Figure 1: Carte de localisation du secteur d'étude. 


\section{RESULTATS}

\section{Typologie des groupements végétaux}

La matrice brute constituée de 156 relevés et de 430 espèces est soumise à une analyse globale de gradient par la DCA. Les trois premiers axes factoriels expliquent 9,75 $\%$ de l'inertie totale. Les résultats de cette analyse ont permis d'identifier huit groupements végétaux (Figure 2).

La discrimination des relevés sur l'axe 1 de la Figure 2 s'est faite suivant les gradients topographique et pédologique (profondeur du sol). De la gauche vers la droite de cet axe, la profondeur et l'humidité $\mathrm{du}$ sol augmentent quand on passe des positions topographiques hautes vers les basses positions sur les toposéquences. L'axe 2 est indicateur de la structure de la végétation. Du haut vers le bas de cet axe, on note une augmentation de la densité des ligneux. La Figure 3 présente l'ordination des groupements identifiés sur le plan des axes 1 et 2 de la CCA. Il ressort de l'analyse de la Figure 3 que les variables testées ont une relation avec les axes canoniques. Le test de Monte Carlo a montré que les variables environnementales testées ont un effet significatif sur la distribution des groupements végétaux. A l'issue des analyses, les huit groupements obtenus sont: - G1: Groupement végétal à Loudetiopsis ambiens et Panicum phragmitoides des dépressions marécageuses; - G2: Groupement végétal à Anchomanes welwitschii et Berlinia grandiflora établi le long des cours d'eau sur des sols profonds et humides et sur les axes de drainage sur des sols hydromorphes; - G3 : Groupement végétal à Andropogon tectorum et Anogeissus leiocarpa des bas de versant et des mi-pentes sur des sols profonds et humides; - G4: Groupement végétal à Andropogon gayanus et Combretum fragrans des bas de versants et mi-pentes sur des sols profonds et humides; - G5: Groupement végétal à Ageratum conyzoides et Tridax procumbens des formations postculturales sur différentes facettes topographiques sur des sols exondés ; - G6 : Groupement végétal à Andropogon schirensis et Isoberlinia tomentosa des interfluves et des mi-pentes sur des sols exondés; - G7 : Groupement végétal à Hyparrhenia involucrata et Pteleopsis suberosa des jachères sur différentes facettes topographiques sur des sols exondés ; - G8 : Groupement végétal à Aframomum alboviolaceum et Uapaca togoensis des bas de versants et des mipentes sur des sols profonds et humides.

L'indice de similitude de Jaccard indique que les groupements végétaux sont dissemblables au seuil de $50 \%$. Cependant, les groupements G6, G7 et G8 sont floristiquement proches, mais nos observations du terrain ont confirmé leur différence sur les plans physionomique et du substrat édaphique sur lequel ils sont établis. Le Tableau 1 présente les caractéristiques floristiques et écologiques des groupements végétaux identifiés.

De l'analyse du Tableau 1, il ressort que les différents groupements végétaux sont caractéristiques des types de biotope. Les facteurs environnementaux interviennent alors significativement dans la discrimination des groupements végétaux des petits bassins versants. Cette discrimination montre que la topographie, la texture, la profondeur et l'humidité du sol sont les facteurs les plus déterminants.

\section{Composition floristique, diversité spécifique et structure des groupements végétaux}

Au total, 430 espèces réparties en 257 genres et 78 familles ont été recensées. Les Leguminosae (83 espèces) et les Poaceae (76 espèces) sont les familles les plus représentées. Le Tableau 2 présente la composition floristique, la diversité spécifique et la structure des groupements végétaux.

Le Tableau 2 indique que les plus faibles valeurs de richesse spécifique sont obtenues dans les formations postculturales et sur les versants des forêts denses sèches alors que les plus fortes sont obtenues dans les forêts claires, les savanes boisées et les forêts galeries présentes le long des cours d'eau sur des sols profonds et humides. 
L'indice de diversité de Shannon et l'Equitabilité de Pielou sont plus élevés à mipente, au bas des versants et dans les talwegs des forêts claires et forêts galeries alors que les plus faibles sont obtenues dans les champs et jachères et dans les dépressions marécageuses. La densité et la surface terrière moyenne des ligneux varient non seulement suivant la profondeur et l'humidité du sol mais aussi selon le type de formation végétale et le degré de perturbation.

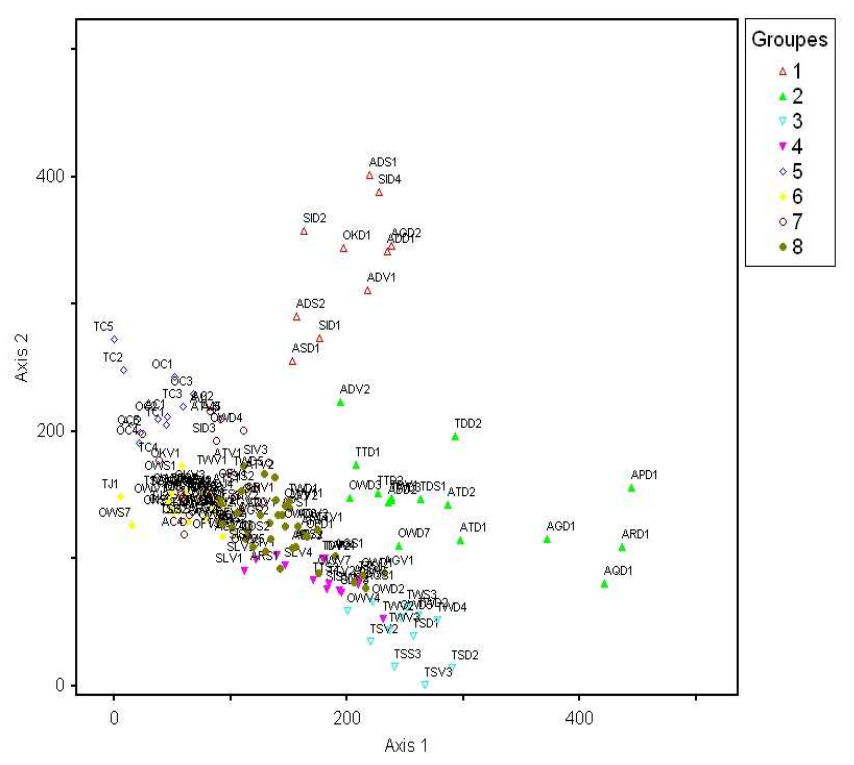

Figure 2 : Ordination des relevés sur le plan des axes 1 et 2 de la DCA.

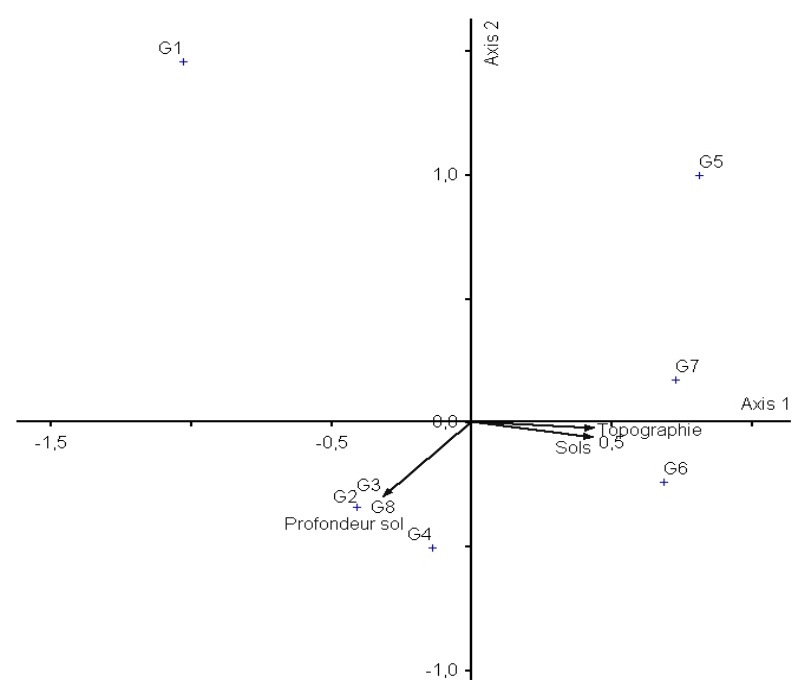

Figure 3 : Répartition des groupements végétaux identifiés et des variables environnementales dans le plan des axes 1 et 2 de la CCA. 
I. TOKO IMOROU / Int. J. Biol. Chem. Sci. 7(6): 2178-2192, 2013

Tableau 1 : Caractéristiques floristiques et écologiques des groupements végétaux.

\begin{tabular}{|c|c|c|c|c|c|c|c|}
\hline Groupements végétaux & Topographie & Types de sol & $\begin{array}{l}\text { Texture des } \\
\text { sols }\end{array}$ & $\begin{array}{c}\text { Profondeur } \\
\text { des sols }\end{array}$ & $\begin{array}{l}\text { Types de } \\
\text { végétation }\end{array}$ & $\begin{array}{l}\text { Deux espèces } \\
\text { abondantes }\end{array}$ & $\begin{array}{l}\text { Autres espèces } \\
\text { caractéristiques }\end{array}$ \\
\hline $\begin{array}{l}\text { G1: Loudetiopsis ambiens } \\
\text { et Panicum phragmitoides }\end{array}$ & $\begin{array}{l}\text { Dépressions } \\
\text { marécageuses }\end{array}$ & Gleyosols & Argileuse & 50 et $70 \mathrm{~cm}$ & $\begin{array}{l}\text { Savanes } \\
\text { herbeuses }\end{array}$ & $\begin{array}{l}\text { Hyparrhenia rufa, } \\
\text { Loudetia arundinacea }\end{array}$ & $\begin{array}{l}\text { Aspilia paludosa, } \\
\text { Hyparrhenia rufa }\end{array}$ \\
\hline $\begin{array}{l}\mathrm{G} 2 \text { : Anchomanes } \\
\text { welwitschii et Berlinia } \\
\text { grandiflora }\end{array}$ & $\begin{array}{l}\text { Talwegs, axes } \\
\text { de drainage }\end{array}$ & $\begin{array}{l}\text { Fluvisols, } \\
\text { gleyosols }\end{array}$ & $\begin{array}{l}\text { Limono- } \\
\text { sableuse, } \\
\text { limono- } \\
\text { argileuse }\end{array}$ & $80-100 \mathrm{~cm}$ & Forêts galeries & $\begin{array}{l}\text { Anogeissus leiocarpa, } \\
\text { Berlinia grandiflora }\end{array}$ & $\begin{array}{l}\text { Manilkara multinervis, } \\
\text { Raphia sudanica }\end{array}$ \\
\hline $\begin{array}{l}\text { G3 : Andropogon tectorum } \\
\text { et Anogeissus leiocarpa }\end{array}$ & $\begin{array}{l}\text { Bas des } \\
\text { versants, mi- } \\
\text { pentes }\end{array}$ & $\begin{array}{l}\text { Lixisols, } \\
\text { acrisols }\end{array}$ & $\begin{array}{l}\text { Limoneuse, } \\
\text { limono- } \\
\text { sableuse, } \\
\text { limono- } \\
\text { argileuse }\end{array}$ & $\geq 90 \mathrm{~cm}$ & $\begin{array}{l}\text { Forêts denses } \\
\text { sèches }\end{array}$ & $\begin{array}{l}\text { Anogeissus leiocarpa, } \\
\text { Andropogon tectorum }\end{array}$ & $\begin{array}{l}\text { Allophylus africanus, } \\
\text { Lonchocarpus } \\
\text { cyanescens }\end{array}$ \\
\hline $\begin{array}{l}\mathrm{G} 4 \text { : Andropogon gayanus } \\
\text { et Combretum fragrans }\end{array}$ & $\begin{array}{l}\text { Bas des } \\
\text { versants, mi- } \\
\text { pentes }\end{array}$ & Gleyosols & $\begin{array}{l}\text { Limono- } \\
\text { argileuse, } \\
\text { argilo- } \\
\text { limoneuse }\end{array}$ & $\begin{array}{l}50 \text { et } 100 \\
\mathrm{~cm}\end{array}$ & $\begin{array}{l}\text { Savanes } \\
\text { arborées } \\
\text { /boisées }\end{array}$ & $\begin{array}{l}\text { Andropogon gayanus, } \\
\text { Andropogon tectorum }\end{array}$ & $\begin{array}{l}\text { Ampelocissus leonensis, } \\
\text { Lonchocarpus sericeus }\end{array}$ \\
\hline $\begin{array}{l}\text { G5 : Ageratum conyzoides } \\
\text { et Tridax procumbens }\end{array}$ & $\begin{array}{l}\text { Bas des } \\
\text { versants, mi- } \\
\text { pentes, } \\
\text { interfluves }\end{array}$ & $\begin{array}{l}\text { Lixisols, } \\
\text { acrisols, } \\
\text { plinthosols }\end{array}$ & $\begin{array}{l}\text { Limono- } \\
\text { sableuse, } \\
\text { limono- } \\
\text { argileuse, sablo- } \\
\text { argileuse, } \\
\text { sablo-graveleuse }\end{array}$ & $10-40 \mathrm{~cm}$ & Jachères & $\begin{array}{l}\text { Ageratum conyzoides, } \\
\text { Vitex doniana }\end{array}$ & $\begin{array}{l}\text { Vicoa leptoclada, } \\
\text { Boerhavia erecta }\end{array}$ \\
\hline
\end{tabular}


I. TOKO IMOROU / Int. J. Biol. Chem. Sci. 7(6): 2178-2192, 2013

\begin{tabular}{|c|c|c|c|c|c|c|c|}
\hline $\begin{array}{l}\text { G6 : Andropogon } \\
\text { schirensis et Isoberlinia } \\
\text { tomentosa }\end{array}$ & $\begin{array}{l}\text { Interfluves, haut } \\
\text { des versants, mi- } \\
\text { pentes }\end{array}$ & Plinthosols & $\begin{array}{l}\text { Sablo-argileuse, } \\
\text { sablo-graveleuse }\end{array}$ & $30-70 \mathrm{~cm}$ & $\begin{array}{l}\text { Forêts claires, } \\
\text { savanes boisées }\end{array}$ & $\begin{array}{l}\text { Isoberlinia doka, } \\
\text { Hyparrhenia smithiana }\end{array}$ & $\begin{array}{l}\text { Monotes kerstingii, } \\
\text { Psorospermum } \\
\text { febrifugum }\end{array}$ \\
\hline $\begin{array}{l}\text { G7 : Hyparrhenia } \\
\text { involucrata et Pteleopsis } \\
\text { suberosa }\end{array}$ & $\begin{array}{l}\text { Bas des } \\
\text { versants, mi- } \\
\text { pentes, } \\
\text { interfluves }\end{array}$ & $\begin{array}{l}\text { Lixisols, } \\
\text { acrisols, } \\
\text { plinthosols }\end{array}$ & $\begin{array}{l}\text { Limono- } \\
\text { sableuse, } \\
\text { limono- } \\
\text { argileuse, sablo- } \\
\text { argileuse, sablo- } \\
\text { graveleuse }\end{array}$ & $30-60 \mathrm{~cm}$ & Jachères & $\begin{array}{l}\text { Hyparrhenia involucrata, } \\
\text { Hyparrhenia smithiana }\end{array}$ & $\begin{array}{l}\text { Lophira lanceolata, } \\
\text { Hyparrhenia } \\
\text { involucrata }\end{array}$ \\
\hline $\begin{array}{l}\text { G8 : Aframomum } \\
\text { alboviolaceum et Uapaca } \\
\text { togoensis }\end{array}$ & $\begin{array}{l}\text { Mi-pente, bas } \\
\text { des versants }\end{array}$ & $\begin{array}{l}\text { Lixisols, } \\
\text { acrisols }\end{array}$ & $\begin{array}{l}\text { Argileuse, } \\
\text { argilo-sableuse, } \\
\text { argilo- } \\
\text { graveleuse }\end{array}$ & $\begin{array}{l}50 \text { et } 100 \\
\mathrm{~cm}\end{array}$ & $\begin{array}{l}\text { Forêts claires, } \\
\text { savanes boisées }\end{array}$ & $\begin{array}{l}\text { Uapaca togoensis, } \\
\text { Isoberlinia doka }\end{array}$ & $\begin{array}{l}\text { Trichilia emetica, } \\
\text { Burkea africana }\end{array}$ \\
\hline
\end{tabular}


Tableau 2 : Composition floristique, diversité spécifique et structure des groupements végétaux.

\begin{tabular}{|c|c|c|c|c|c|c|c|c|c|c|c|c|}
\hline \multirow{2}{*}{ Groupements végétaux } & \multirow[b]{2}{*}{$\begin{array}{l}\text { Nbre de } \\
\text { relevés }\end{array}$} & \multicolumn{3}{|c|}{ Nombre d'espèces } & \multirow{2}{*}{$\begin{array}{c}\text { Nbre } \\
\text { de } \\
\text { genres }\end{array}$} & \multirow{2}{*}{$\begin{array}{l}\text { Nbre de } \\
\text { familles }\end{array}$} & \multirow[t]{2}{*}{$\mathbf{R}$} & \multirow[t]{2}{*}{ H (bits) } & \multirow[t]{2}{*}{$\mathbf{E}$} & \multirow{2}{*}{$\begin{array}{c}\text { D } \\
\text { (tiges.ha }^{-1)}\end{array}$} & \multirow[t]{2}{*}{$\mathbf{G}\left(\mathbf{m}^{2} \cdot \mathbf{h a}^{-1)}\right.$} & \multirow[t]{2}{*}{$\mathbf{B v}\left(\mathbf{m}^{3} / \mathbf{h a}\right)$} \\
\hline & & Ligneuses & Herbacées & Total & & & & & & & & \\
\hline $\begin{array}{l}\text { G1: } L . \text { ambiens et } P \\
\text { phragmitoides }\end{array}$ & 10 & 30 & 92 & 122 & 94 & 41 & $34 \pm 14$ & $1,88 \pm 1,17$ & $0,77 \pm 0,25$ & $270 \pm 193$ & $4,05 \pm 1,82$ & $\begin{array}{l}15105,20 \pm \\
4161,60\end{array}$ \\
\hline $\begin{array}{l}\mathrm{G} 2: A \text { welwitschii et } B \\
\text { grandiflora }\end{array}$ & 16 & 100 & 121 & 221 & 163 & 57 & $42 \pm 14$ & $3,46 \pm 0,71$ & $0,88 \pm 0,08$ & $668 \pm 224$ & $19,44 \pm 11,11$ & $\begin{array}{l}6204,94 \quad \pm \\
4673,93\end{array}$ \\
\hline $\begin{array}{l}\mathrm{G} 3: \text { A tectorum et } A \\
\text { leiocarpa }\end{array}$ & 12 & 50 & 63 & 113 & 86 & 33 & $27 \pm 8$ & $2,25 \pm 0,86$ & $0,71 \pm 0,10$ & $637 \pm 255$ & $17,71 \pm 8,62$ & $\begin{array}{ll}5386,63 \quad \pm \\
2807,45\end{array}$ \\
\hline $\begin{array}{l}\mathrm{G} 4: \text { A gayanus et } C \\
\text { fragrans }\end{array}$ & 14 & 68 & 85 & 153 & 109 & 44 & $42 \pm 10$ & $3,28 \pm 0,90$ & $0,84 \pm 0,09$ & $814 \pm 288$ & $14,17 \pm 9,90$ & $\begin{array}{l}11994,29 \pm \\
2325,79\end{array}$ \\
\hline $\begin{array}{l}\text { G5 : A conyzoides et } T \\
\text { procumbens }\end{array}$ & 12 & 35 & 81 & 116 & 92 & 35 & $27 \pm 10$ & $1,40 \pm 0,97$ & $0,67 \pm 0,41$ & $104 \pm 60$ & $3,21 \pm 2,01$ & $\begin{array}{ll}1917,08 \quad \pm \\
652,35\end{array}$ \\
\hline $\begin{array}{l}\text { G6 : Aschirensis et I } \\
\text { tomentosa }\end{array}$ & 28 & 69 & 127 & 196 & 132 & 46 & $48 \pm 7$ & $3,69 \pm 0,46$ & $0,85 \pm 0,07$ & $772 \pm 266$ & $12,43 \pm 4,55$ & $\begin{array}{l}5603,89 \pm \\
3669,25\end{array}$ \\
\hline $\begin{array}{l}\text { G7 : } H \text { involucrata et } P \\
\text { suberosa }\end{array}$ & 26 & 78 & 145 & 223 & 188 & 52 & $39 \pm 12$ & $2,84 \pm 1,00$ & $0,84 \pm 0,19$ & $426 \pm 353$ & $7,12 \pm 5,07$ & $\begin{array}{ll}9615,33 \quad \pm \\
6992,85\end{array}$ \\
\hline $\begin{array}{l}\text { G8 : A alboviolaceum et } \\
U \text { togoensis }\end{array}$ & 38 & 102 & 159 & 261 & 178 & 56 & $51 \pm 12$ & $3,88 \pm 0,44$ & $0,85 \pm 0,07$ & $1007 \pm 370$ & $18,01 \pm 6,50$ & $\begin{array}{ll}4793,51 \quad \pm \\
4713,53\end{array}$ \\
\hline
\end{tabular}

$\mathrm{R}$ : Richesse spécifique, H : Indice de diversité de Shannon, E : Equitabilité de Pielou, D : Densité des ligneux, G : Surface terrière moyenne, Bv : Biovolume de la strate herbacée 


\section{DISCUSSION}

Facteurs déterminant la répartition spatiale des groupements végétaux

Les facteurs environnementaux interviennent de manière significative dans la discrimination des groupements végétaux des petits bassins versants. La topographie, la texture, la profondeur et 1'humidité du sol sont les facteurs les plus déterminants dans la distribution des groupements végétaux. Toutefois, la profondeur du sol est le facteur le plus important. De nombreux auteurs (Grouzis, 1988; Wittig et al., 2000 ; Parmentier et al., 2001; Parmentier, 2003 ; Oumorou, 2003; Wala, 2004; Ouedraogo, 2006; Sesnie et al., 2009; Toko et al., 2010 ; Toko et Sinsin, 2011) ont également distingué des communautés végétales sur la base d'unités morphopédologiques. Par contre, les résultats de cette étude montrent que la topographie à elle seule ne détermine pas la répartition des formations végétales. En effet, à une même position topographique, peuvent correspondre des formations différentes. Par exemple, en dehors des dépressions qui abritent uniquement les savanes herbeuses et le long des rivières où s'établissent essentiellement les forêts galeries, les autres formations végétales se retrouvent sur différentes facettes topographiques. Ainsi, les savanes boisées sont présentes sur les hauts de versant ou au bas des versants. Sur les interfluves s'établissent les forêts claires ou les savanes arborées. D'autres auteurs notamment Oumorou (2003), Wala (2004), Orthmann (2005) ont révélé l'absence de relation linéaire entre la répartition spatiale de la végétation et la topographie.

\section{Rôle des facteurs écologiques sur la composition floristique et la diversité spécifique}

Les sols profonds et humides des dépressions et des bas de versant, de par l'importance de leur réserve en eau, favorisent la germination et la croissance des herbacées. Sur ces types de sols, les herbacées ne souffrent d'aucune limitation et elles assurent de façon continue leur alimentation hydrique et minérale. Par contre, sur les sols secs et peu profonds des interfluves et des hauts de versant, le point de flétrissement est vite atteint, limitant de facto la bonne croissance et l'abondance des herbacées. La richesse spécifique quant à elle est fortement corrélée avec les variables pédologiques et topographiques. La richesse spécifique évolue avec la profondeur du sol, tandis qu'elle baisse avec l'augmentation de la pente. Les plus fortes valeurs de la richesse spécifique sont obtenues dans les formations présentes le long des cours d'eau, sur les drains et au bas des versants. La forte valeur de richesse spécifique obtenue dans les basses positions topographiques est liée à la profondeur et à l'humidité permanente des sols. La présence de cours d'eau le long des galeries forestières maintient le niveau d'humidité du sol de ces formations, ce qui entretient un microclimat humide qui favorise l'installation et le développement de nombreuses espèces. Nombre d'auteurs notamment Clark et al. (1995), Wezel et Bocker (1998), Oumorou (2003), Wala (2004), Bauer et Peterson (2005), Orthmann (2005), Djego et Sinsin (2006), Bohlman et al. (2008), Sesnie et al. (2009) ont également mis en exergue les conditions édaphiques et topographiques dans la répartition spatiale des espèces.

Les faibles valeurs de diversité sont obtenues dans les groupements végétaux où les conditions édaphiques, l'insolation ou la pression anthropique constituent des facteurs limitants. Les conditions édaphiques expliquent la plus grande part de la variance de la diversité des espèces. Ainsi, la faible épaisseur et le rajeunissement des sols dans les champs et les jeunes jachères suite à la fréquence des labours et du sarclage sont la cause de la faible diversité floristique. Ces actions mettent à nus les sols en les appauvrissant en matière organique ; ce qui a pour conséquence une faible diversité caractérisé par une forte proportion d'espèces messicoles et ségétales. Par ailleurs, le taux de 
recouvrement de la strate arborescente dans les forêts denses sèches fait que les rayons solaires ne parviennent pas au sol. Ainsi, la faible diversité du groupement végétal à Andropogon tectorum et Anogeissus leiocarpa établi dans les forêts denses sèches est due à l'abondance et la dominance de Anogeissus leiocarpa. L'ombrage de cette espèce réduit la quantité de lumière solaire qui parvient à la strate herbacée. Ce faible éclairement limite les activités photosynthétiques et retarde la décomposition de la litière, empêchant de facto la germination des diaspores. La lumière solaire est donc une composante principale de la productivité des formations végétales soudaniennes (Semmartin et Oesterheld, 1996 ; Altesor et al., 2005 ; Djego et Sinsin, 2006 ; Toko et Sinsin, 2011).

Par ailleurs, la pratique des activités pastorales a contribué à l'augmentation de la diversité dans le groupement à Andropogon schirensis et Isoberlinia tomentosa établi sur des sols secs. En effet, les activités pastorales qui s'y déroulent, contribuent à la réduction du biovolume de la strate herbacée ce qui atténue l'intensité des feux de végétation et favorise l'émergence des espèces. Par ailleurs, les déjections des troupeaux de bovins améliorent la fertilité des sols, ce qui augmente la richesse floristique en espèces nitrophiles. Ces résultats obtenus corroborent ceux de Scholes et Archer (1997), Sanderson et al. (2004), Altesor et al. (2005), de Bello et al. (2007) et Hendricks et al. (2007) qui ont estimé que la pratique des activités pastorales dans un écosystème contribue à l'augmentation de la diversité en termes de richesse spécifique et d'abondance relative des espèces.

En outre, la grande profondeur et l'humidité permanente des sols font que le groupement à Anchomanes difformis et Berlinia grandiflora et le groupement à Aframomum alboviolaceum et Uapaca togoensis sont favorables à l'installation de nombreuses espèces. Toutefois, l'abondance de l'eau constitue un facteur limitant pour la diversité spécifique. Dans les dépressions marécageuses et sur les axes de drainage, l'engorgement des sols empêche l'oxygénation des plantes, ce qui fait que les espèces mésophiles et les espèces peu adaptées à ces biotopes meurent par asphyxie. Ainsi, ces biotopes sont caractérisés par la prédominance d'un petit nombre d'espèces notamment Terminalia macroptera et Mitragyna inermis qui dominent les groupements hygrophiles boisés qu'on retrouve dans les lits des ruisseaux et qui sont peu diversifiés à cause de l'engorgement permanent du sol et $\mathrm{du}$ fort taux de recouvrement des ligneux.

\section{Relation entre la structure de la végétation et les conditions édaphiques \\ Les variables environnementales} testées ont montré que la densité et la surface terrière des ligneux varient selon la topographie et les conditions édaphiques (texture, profondeur et humidité du sol). Ainsi, 1'inondation quasi permanente des sols des dépressions marécageuses est un facteur limitant pour le développement des espèces autres que les espèces hydrophiles notamment Terminalia macroptera et Mitragyna inermis qui parviennent à résister à l'asphyxie. Par contre, les bonnes conditions édaphiques caractérisées par la profondeur, l'abondance de la litière, la texture limoneuse ou argilolimoneuse et 1 'humidité des sols conduisent à une forte densité et à une grande surface terrière des ligneux. L'impact des activités anthropiques telles que l'agriculture, les feux de végétation, le surpâturage et l'exploitation forestière sur les formations naturelles est perceptible à travers les faibles valeurs de densité et de surface terrière des ligneux. Plusieurs auteurs sont parvenus à la même conclusion selon laquelle, la structure de la végétation est d'une part sous la dépendance des conditions édaphiques puisque étroitement dépendante de la nature et de la disponibilité de l'eau dans le sol et, d'autre part, de l'intensité des activités anthropiques (Akoegninou et Akpagana, 1997 ; Hansen et al., 2000; Tenté, 2000 ; Mappin et al., 2003; 
Devineau et Fournier, 2005; Thornes, 2005; Gould et al., 2006; Ouedraogo et al., 2008; Arouna et al., 2010 ; Toko et al., 2010 ; Toko Imorou et al., 2010; Arouna et al., 2011; Toko Mouhamadou et al., 2012; Toko Mouhamadou et al., 2013 ; Houessou et al., 2013).

\section{REMERCIEMENTS}

Nous remercions les projets BIOTA et IMPETUS qui ont financé cette recherche.

\section{REFERENCES}

Adomou AC, Sinsin B, van der Maesen LJG. 2006. Phytosociological and chorological approaches to phytogeography: a mesoscale study in Benin. Syst. Geogr. P1., 76: 155-178.

Akoegninou A, Akpagana K. 1997. Etude cartographique et dynamique de la végétation de l'aire classée de la colline de Savalou (Bénin). J. Bot. Soc. Bot. Fr., 3: 69-81.

Altesor A, Oesterheld M, Leoni E, Lezama F, Rodriguez C. 2005. Effect of grazing on community structure and productivity of a Uruguayan grassland. Plant Ecology, 179: 83-91.

Arouna O, Toko Imorou I, Djogbenou CP, Sinsin B. 2010. Impact de la dynamique spatio- temporelle de l'occupation du sol sur la végétation en zone soudanoguinéenne au Bénin. Rev. Sc. Env. Univ., Lomé (Togo), 006 : 161-186.

Arouna O, Toko I, Djogbenou CP, Sinsin B. 2011. Comparative analysis of local populations' perceptions of socioeconomic determinants of vegetation degradation in sudano-guinean area in Benin (West Africa). International Journal of Biodiversity and Conservation, 3(7): 327-337.

Bauer JT, Peterson AT. 2005. Visualizing environmental correlates of species geographical range limits. Diversity and Distributions, 11: 275-278.

de Bello F, Lepš J, Sebastià MT. 2007. Grazing effects on the species-area relationship : Variation along a climatic gradient in NE Spain. Journal of Vegetation Science, 18: 25-34.

Bohlman SA, Laurance WF, Laurance SG, Nascimento HEM, Fearnside PM, Andrade A. 2008. Importance of soils, topography and geographic distance in structuring central Amazonian tree communities. Journal of Vegetation Science, 19: 863-874.

Clark DA, Clark DB, Sandoval R, Castro MV. 1995. Edaphic and human effects on landscape-scale distributions of tropical rain forest palm. Ecology, 76: 2581-2595.

Devineau JL, Fournier A. 2005. To what extent can simple plant biological traits account for the response of the herbaceous layer to environmental changes in fallow-savanna vegetation (West Burkina Faso, West Africa)? Flora, 200: 361-375.

Djego J, Sinsin B. 2006. Impact des espèces exotiques plantées sur la diversité spécifique des phytocénoses de leur sous-bois. Systematics and Geography of Plants, 76: 191-209.

Dufrene M, Legendre P. 1997. Species Assemblages and Indicator Species: The Need for a Flexible Asymmetrical Approch. Ecological Monographs, 67(3): 345-366.

Faure P. 1977. Carte pédologique de reconnaissance de la république populaire du Bénin à 1/200.000, Feuille de Djougou. ORSTOM, Paris.

Gould WA, Gonzalez G, Carrero Rivera G. 2006. Structure and composition of vegetation along an elevational gradient in Puerto Rico. Journal of Vegetation Science, 17: 653-664.

Grouzis M. 1988. Structure, productivité et dynamique des systèmes écologiques sahéliens (Mare d'Oursi, Burkina Faso). ORSTOM, Paris.

Hansen AJ, Rotella JJ, Kraska MPV, Brown D. 2000. Spatial patterns of primary productivity in the Greater Yellowstone 
Ecosystem Landscape. Ecology, 15: 505522.

Hendricks HH, Bond WJ, Midgley JJ, Novellie PA. 2007. Biodiversity conservation and pastoralism-reducing herd size in a communal livestock production system in Richtersveld National Park. Journal of Arid Environments, 70: 718-727.

Houessou LG, Teka O, Toko Imorou I, Lykke AM, Sinsin B. 2013. Land Use and LandCover Change at "W" Biosphere Reserve and Its Surroundings Areas in Benin Republic (West Africa). Environment and Natural Resources Research, 3(2): 87-101.

Houinato MRB, Sinsin B. 2001. Analyse phytogéographique de la région des Monts Kouffé au Bénin. XVIth AETFAT Congress. Syst. Geogr. Pl., 71: 889-910.

Hutchinson J, Dalziel JM. 1954, 1958, 1963, 1968, 1972. Flora of West Tropical Africa, (vol. 1 à 3). Crown Agents for Overseas Governments and Administrations: Londres; Vol. 1, tomes 1 \& 2, 828 p., Vol. 2, 544 p., Vol. 3, tomes $1 \& 2,852 \mathrm{p}$.

Lebrun JP, Stock AL. 1991-1997. Enumérations des plantes à fleurs d'Afrique tropicale. Editions des Conser. et Jard. Bot. Génève, Vol. I-IV.

Mappin KA, Pate JS, Bell TL. 2003. Productivity and water relations of burnt and long-unburnt semi-arid shrubland in Western Australia. Plant and Soil, 257: 321-340.

McCune B, Mefford MJ. 1999. Pc-ord. Multivariate analysis of ecological data, version 5. MjM Software Design, Gleneden Beach, OR, US.

Økland RH, Eilertsen O. 1994. Canonical correspondence analysis with variation partitioning: some comments and an application. Journal of Vegetation Science 5: 117-126.

Orekan VOA, 2007. Implementation of the local land-use and land-cover change model CLUE-s for Central Benin by using socio-economic and remote sensing data. Dissertation, University of Bonn, Bonn, Germany, 230 p.

Orthmann B. 2005. Vegetation ecology of a woodland-savanna mosaic in central Benin (West Africa): Ecosystem analysis with a focus on the impact of selective logging Dissertation. University of Rostock, Rostock, Germany, 148 p.

Ouedraogo O, Thiombiano A, Hahn-Hadjali K, Guinko S. 2008. Diversité et structure des groupements ligneux du parc national d'Arly (Est du Burkina-Faso). Flora et Vegetatio Sudano-Sambesica, 11: 5-16.

Ouedraogo A. 2006. Diversité et dynamique de la végétation ligneuse de la partie orientale du Burkina Faso. Thèse de doctorat. Université de Ouagadougou, Ouagadougou, Burkina-Faso, 230 p.

Oumorou M, Sinsin B, Lejoly J. 1998. Etude phytosociologique de deux faciès à Hyparrhenia involucrata Stapf dans les savanes soudaniennes du Bénin. XXVIII Colloques phytosociologiques. Vegetazione postglaciale passata e presente. Camerino, 1054-1071.

Oumorou M. 2003. Etude écologique, floristique, phytogéographique et phytosociologique des inselbergs du Bénin. Thèse de doctorat. Université Libre de Bruxelles, Bruxelles, Belgique, 210 p + annexes.

Parmentier I, Lejoly J, Nguema N. 2001. La végétation des inselbergs de Piedra Nzas (Guinée Equatoriale Continentale). Acta Botanica Gallica, 148(4): 341-365.

Parmentier I. 2003. Study of the vegetation composition in three inselbergs from Continental Equatorial Guinea (western central Africa): Effects of site, soil factors and position relative to forest fringe. Belgian Journal of Botany, 136(1): 63-72.

Parmentier I, Stevart T, Hardy OJ. 2005. The inselberg flora of Atlantic Central Africa. I. Determinants of species assemblages. Journal of Biogeography, 32: 685-696. 
Pisces Conservation LTD. 2002. Community Analysis Package (CAP), a program to search for structure in ecological community data, version 2.0. Pennington, England, IRC House.

Russel-Smith J. 1991. Classification, species richness and environmental relations of monsoon rain forest in northern Australia. Journal of Vegetation Science, 2: 259278.

Sanderson fMA, Skinner RH, Barker DJ, Edwards GR, Tracy BF, Wedin DA. 2004. Plant species diversity and management of temperate forage and grazing land ecosystems. Crop Science, 44: 1132-1144.

Scholes RJ, Archer SR. 1997. Tree-grass interactions in savannas. Ann. Rev. Ecol. Syst., 28: 517-544.

Semmartin M, Oesterheld M. 1996. Effect of grazing pattern on primary productivity. Oikos, 75: 431-436.

Sesnie SE, Finegan B, Gessler PE, Ramos Z. 2009. Landscape-scale environmental and floristic variation in Costa Rican oldgrowth rain forest remnants. Biotropica, 41(1): 16-29.

Sinsin B. 1994. Individualisation et hiérarchisation des Phytocénoses soudaniennes du nord-Bénin. Bel. Journ. Bot., 127 (1) : 87-103.

Sinsin B, Omorou M, Ogoubyi V. 1996. Les faciès à Andropogon pseudapricus des groupements post-culturaux et des savanes arbustives du Nord-Bénin : dissemblance floristique et caractères communs. In The Biodiversity of African Plants, van der Maesen LJG, van der Burgt \& van Medenbach de Rooy (eds). Kluwer Academic Publishers: Netherlands; 231-238.

Sinsin B. 1999. Description floristique, structurale et écologique des associations végétales du domaine soudanien du nordBénin. Documents Phytosociologiques. N.S. 10: 311-335.

Sinsin B, Oumorou M. 2000. Etude de la diversité spécifique du groupement à
Cochlospermum tinctorium A. Rich. des savanes arbustives du nord-Bénin. Acta Bot. Gallica, 147 (4) : 345-360.

Sinsin B. 2001. Formes de vie et diversité spécifique des associations de forêts claires $\mathrm{du}$ nord $\mathrm{du}$ Bénin. XVIth AETFAT Congress. Syst. Geogr. Pl, , 71: 873-888.

Sinsin B, Wotto J. 2003. Changes in floristic composition of grazing land in northern Sudanian zone (Benin). In Rangelands in the new millennium, Allsopp $\mathrm{N}$, Palmer AR, Milton SJ, Kirkman KP, Kerly GIH, Hurt CR, Brown CJ (eds). VII ${ }^{\text {th }}$ International Rangeland Congress, Durban South Africa, 26 July - 1 August 2003, pp 402-404. ISBN 0-958-45348-9. African Journal of Range \& Forage Science, 20(2): 89-100.

Tchamie T, Bouraïma M. 1997. Les formations végétales du plateau SoudouDako dans la chaîne de l'Atakora et leur évolution récente (Nord Togo). J. Bot. Soc. Fr., 3: 83-94.

Tente BA. 2000. Dynamique actuelle de l'occupation du sol dans le massif forestier de l'Atacora: secteur PermaToucountouna. Mémoire de D.E.A, FLASH, UNB, Abomey-Calavi, Bénin, $82 \mathrm{p}$.

Thornes JB. 2005. Coupling erosion, vegetation and grazing. Land Degrad. Develop., 16: 127-138.

Toko I, Orthmann B, Porembski S, Sinsin B. 2010. Facteurs déterminant la répartition spatiale de la flore et de la structure de la végétation dans les sous bassins d'Aguimo et de Térou-Wannou au centre du Bénin. Rev. Sc. Env. Univ., Lomé (Togo), 006 : 5-24.

Toko Imorou I, Arouna O, Sinsin B. 2010. Cartographie des changements spatiotemporels de l'occupation du sol de la forêt classée de l'Alibori Supérieur au Nord-Bénin. BenGéo., 7 : 22-39.

Toko Mouhamadou I, Toure F, Toko Imorou I, Sinsin B. 2012. Indices de structures spatiales des îlots de forêts denses dans 
la région des Monts Kouffé. VertigO, 12(3): 1-17.

Toko Mouhamadou I, Toko Imorou I, Gbegbo MC, Sinsin B. 2013. Structure et composition floristiques des forêts denses sèches de la région des Monts Kouffé au Bénin. Journal of Applied Biosciences, 64: 4787-4796.

Thiombiano A. 2000. Les particularités de la végétation et de la flore de la chaîne du Gobnangou dans le Sud-Est du Burkina Faso. Etude Flor. Vég. Burkina-Faso, 5: 49-64.

Wittig R, Hahn-Hadjali K, R: Richesse spécifique, $\mathrm{H}$ : Indice de diversité de Shannon, E : Equitabilité de Pielou, D : Densité des ligneux, G : Surface terrière moyenne, Bv: Biovolume de la strate herbacée.
Wala K. 2004. La végétation de la chaîne de l'Atakora au Benin : diversité floristique, phytosociologie et impact humain. Thèse de doctorat. Université de Lomé, Lomé, Togo, 140 p.

Weber HE, Moravec J, Thervillat JP. 2000. International Code of Phytosociological Nomenclature. $3^{\text {rd }}$ Edition. Journal of Vegetation Science, 11: 739-768.

Wezel A, Boecker R. 1998. Fallow plant communities and site characteristics in semi-arid Niger, West Africa. Journal of Arid Environments, 40: 269-280.

White F. 1983. The vegetation of Africa. A descriptive memoir to accompany the UNESCO/AETFAT/UNSO vegetation map of Africa. UNESCO, Paris. 